\title{
Optimization of Graft Polymerization and Performance of Carboxymethyl Chitosan / Polyacrylamide Flocculants
}

\author{
Emad Ali SOLIMAN ${ }^{1}$, El-Sayed Mohamed El-Sayed MANSOUR ${ }^{2}$, Hammed \\ Ahmed Mohamed HASSAN ${ }^{3}$ and Noha Amaly Mohammed HASSAN ${ }^{4}$ \\ ${ }^{1,4}$ Department of Polymeric Materials Research, Advanced Technology and New Materials Re- \\ search Institute, SRTA-City, New Borg El-Arab City 21934, Alexandria, Egypt \\ ${ }^{2,3}$ Department of Chemistry, Faculty of Science, Alexandria University, El-Shatby, Alexandria 2455, \\ Egypt
}

Correspondence should be addressed to: Emad A. Soliman; emadsoliman@mucsat.sci.eg

Received date: 24 March 2014; Accepted date: 8 April 2014; Published date: 25 July 2014

Academic Editor: Sumit Mishra

Copyright (C) 2014. Ali SOLIMAN, El-Sayed Mohamed El-Sayed MANSOUR, Hammed Ahmed Mohamed HASSAN and Noha Amaly Mohammed HASSAN. Distributed under Creative Commons CC-BY 3.0

\begin{abstract}
In this study, amphoteric graft copolymer-based flocculants were prepared by conventional peroxy graft copolymerization of acrylamide (AM) onto carboxymethyl chitosan (CMCH) using potassium persulphate (PPS) initiator. The optimization of grafting of AM onto $\mathrm{CMCH}$ was carried out by studying the influence of various process parameters including $\mathrm{CMCH}$, acrylamide and PPS concentration, as well as reaction temperature and time on percent grafting. FTIR spectral and thermal analyses of polyacrylamide/carboxymethyl chitosan graft copolymer (CMCH- $g$-PAM) were studied to furnish evidence of grafting. By performing the grafting process via reacting carboxymethyl chitosan $(2 \%)$, acrylamide $(6 \%)$ in presence of PPS $(0.1 \%)$, highest grafting percentage was found at reaction temperature of $55^{\circ} \mathrm{C}$ with reaction time of three hours. The flocculation of the kaolinite suspensions was investigated by studying the effect of CMCH-g-PAM dosage, treatment time and $\mathrm{pH}$ of suspensions. The graft co-polymer flocculants resultant in this study exhibited higher efficiency comparing with those obtained from homo-polymers of carboxymethyl chitosan or polyacrylamide. Furthermore, the flocculation efficiency of these grafted copolymers was significantly increased with increasing the grafting percentage, where the maximum efficiency (93\%) was attained at the highest grafting percentage (146\%).
\end{abstract}

Keywords: Carboxymethyl chitosan-g-polyacrylamide; Suspended kaolinite; Amphoteric flocculants.

\section{Introduction}

Water pollution is owing to all human activities including domestic, industrial and agri- cultural and thus, the causes of pollution in water can be considered endless. However,

Cite this Article as: Emad Ali SOLIMAN, El-Sayed Mohamed El-Sayed MANSOUR, Hammed Ahmed Mohamed HASSAN and Noha Amaly Mohammed HASSAN (2014), "Optimization of Graft Polymerization and Performance of Carboxymethyl Chitosan / Polyacrylamide Flocculants", Journal of Research \& Developments in Chemistry, Vol. 2014 (2014), Article ID 351498, DOI: 10.5171/2014. 351498 
manufacturing plants is a major cause of water pollution that carries away their waste that can contain various dissolved or suspended harmful and toxic substances in fresh water bodies. Suspended substances, nutrients and organic load as chemical oxygen demand (COD) contribute major pollutants in these bodies. Therefore, the removal of these contaminants in waste water is one of the fundamental targets in waste treatment [1]. Further, stringent regulations are releasing in recent years to prevent discharge of these wastes into the municipal sewerage system without any treatment.. Many technologies are developed to treat various types of wastes at adequate levels. However, the economic performance, including investment costs and operation of conventional waste treatment are prohibitive for a variety of industrial simulation, particularly small-scale sector. In this case, the physical-chemical coagulation-flocculation process is a most used key step in the primary purification of industrial wastewater and effluents and in some cases in secondary and tertiary treatment [2].

Generally, such solid-liquid separation processes are carried out by using inorganic or organic coagulating agents. The most common extensively used inorganic coagulants are alum and iron salts. The multivalent characteristics of these cations strongly attract to the colloidal particles rendering them insoluble. On the other hand, organic coagulating agents are also widely used such as polyelectrolyte (cationic or anionic), polyampholytes and nonionic polymers are more efficient agents than their inorganic counterparts and thus, they don't produce voluminous and gelatinous flocs [3]. Surface-adsorbed cationic and anionic polyelectrolytes or polyampholytes promote suspended particle coagulation rates by two distinct mechanisms: charge neutralization and/or polymer bridging [4-5].

Although the synthetic polymers can be tailored to the needs of a particular application and perform well in flocculation, they are often associated with environmental pollution. Natural polymers have the advantages of low cost and biodegradability, but the biodegradability will affect the storage life and the flocculation performance. Therefore, several attempts have been made to combine the best properties of both by grafting synthetic polymers onto the backbone of natural polymers [6]. One of the advantages gained is the consequent reduced biodegradability because of a drastic change in the original regular structure of the natural polymer as well as the increased non-degradable synthetic polymer content within the product. However, the grafting of shear degradable polymers onto a rigid polysaccharide backbone was found to produce fairly shear-stable systems [8-9].

Chitosan is a cationic linear pseudo-natural polymer obtained from $\mathrm{N}$-deacetylation of chitin in alkaline media. Chitosan has several intrinsic properties such as biocompatibility, biodegradability and its degradation products are non-toxic, as well as, its outstanding chelating behavior and thus it directed to numerous applications in waste water treatment, chemical industry, pharmacy, biotechnology and biomedicine [9-13]. Chitin and chitosan are widely used for waste water treatment for the removal both of dispersed particulates and dissolved pollutants. Further, water treated with these polymers experimentally showed a decrease in COD, total nitrogen and microbial population [14-15]. However, acidic solubility, low thermal and mechanical stability and flexible behavior of chitosan are the stumbling blocks in broadening its application in water treatment. Many physical and chemical modification approaches have been developed to jump over these blocks. Recently, a lot of attention has been paid to chemical modification. Though many researchers have shown that primary derivatization followed by graft copolymerization was one of the promising methods to improve tremendously chitosan properties, particularly, via amalgamation of synthetic polymers [16-21]. Grafting flexible polyacrylamide chains onto rigid polysaccharides such as chitosan has been suggested to have a potential to develop efficient, shear-stable, and controlled biodegradable flocculants for the treatment of industrial and mineral processing effluents [22]. In the

Emad Ali SOLIMAN, El-Sayed Mohamed El-Sayed MANSOUR, Hammed Ahmed Mohamed HASSAN and Noha Amaly Mohammed HASSAN (2014), Journal of Research \& Developments in Chemistry, DOI: $10.5171 / 2014.351498$ 
present study, carboxymethyl chitosan ( $\mathrm{CMCH}$ ) was chosen as polysaccharide backbone with polyacrylamide as a graft polymer for the preparation of amphoteric floccu

lants. Whilst chitosan $(\mathrm{CH})$ is a cationic polyelectrolyte, carboxyalkyl chitosan can be considered a polyampholyte which carry positive and negative charges [23]. Thus, the synthesis of graft copolymer was carried out using PPS-induced free radical polymerization. The effect of concentration of $\mathrm{CMCH}, \mathrm{AM}$ and PPS as well as the reaction temperature and time on the graft copolymerization was studied by determining the grafting parameters. Moreover, the flocculation performance of the obtained grafted copolymers was investigated by studying the effects of $\mathrm{pH}$ values, flocculation time and dosage of the flocculants on treating kaolinite suspensions.

\section{Materials and Methods}

\section{Materials}

Chitosan (DD $=85 \%)$, potassium persulphate (PPS), chloroacetic acid, acetic acid (99\%), acrylamide (monomer), and glutaraldehyde $(60 \%)$ were obtained from Sigma-Aldrich chemical LTD (Germany). Acetone (99.9\%), sodium hydroxide, was purchased from El-Nasr pharmaceutical company for chemicals (Egypt). Kaolinite (Egyptian highly refined ball clay powder with very fine size) used mainly for manufacturing refractory bricks and mortar, was kindly donated from Alexandria Company for Refractories.

\section{Preparation of carboxymethyl chitosan (CMCH)}

Carboxymethyl chitosan was prepared according to the method reported by Chen and Park [24]. Briefly $10 \mathrm{~g}$ of chitosan were dispersed in $100 \mathrm{ml}$ of aqueous isopropyl alcohol (50\%) with stirring at room temperature for $30 \mathrm{~min}$. Aliquot portions of 15 $\mathrm{ml}$ sodium hydroxide $(10 \mathrm{M})$ were added at an interval of 5 min under agitation at room temperature. The reaction mixture was stirred for an additional $45 \mathrm{~min}$. Then, $30 \mathrm{~g}$ of chloroacetic acid was dissolved in this mixture portion wise at interval of $5 \mathrm{~min}$ under warming at $55-60{ }^{\circ} \mathrm{C}$ with stirring for $1 \mathrm{~h}$. The resultant solution was filtered. The filtrate was washed with aqueous solution of ethanol $(80 \% v / v)$, then dried in a vac

uum oven at $50{ }^{\circ} \mathrm{C}$ for $24 \mathrm{hrs}$.

\section{Preparation of carboxymethyl chitosan polyacrylamide graft copolymers (CMCH-g-PAM)}

Definite amounts (0.3 -24 gm) of $\mathrm{CMCH}$ were dissolved in $45 \mathrm{ml}$ distilled water, then (0.05 - $1 \mathrm{gm})$ PPS dissolved in $10 \mathrm{ml}$ distilled water then slowly added to the three necked flask to initiate graft copolymerization. In a constant temperature, water bath was maintained at a constant temperature in the range of $25-60{ }^{\circ} \mathrm{C}$. Nitrogen gas was bubbled for $30 \mathrm{~min}$ to remove the dissolved oxygen under stirring. After $10 \mathrm{~min}$, a predetermined amount (1 - 6 gm) of acrylamide dissolved in $45 \mathrm{ml}$ distilled water was charged into a three necked round bottom flask. After grafting time (1-5 hrs), CMCH- $g$-PAM was precipitated by the addition of an excess of acetone (80 \%), filtered, and then the precipitate was washed by aqueous ethanol solution $(90 \% \mathrm{v} / \mathrm{v})$ to eliminate all of $\mathrm{CMCH}, \mathrm{AM}$ and its homopolymer. Afterwards, graft copolymers were dried under vacuum at $50{ }^{\circ} \mathrm{C}$. The grafting percentage was calculated using the following equation;

$$
\% \mathrm{G}=\left[\mathrm{W}_{\text {СMCH-g-PAM }} / \mathrm{W}_{\text {СMCH }}\right] \times 100 \%
$$

\section{FTIR spectroscopic analysis}

FTIR spectra were obtained by a Shimadzu AIM8400 FTIR spectrometer equipped with ATR-8400M objective (Shimadzu Co, Ltd, Tokyo, Japan). This objective adopted a germanium prism tip. The spectra were recorded over a range of $500-4000 \mathrm{~cm}^{-1}$ with a resolution of $2 \mathrm{~cm}^{-1}$ and averaged over 32 scans.

Emad Ali SOLIMAN, El-Sayed Mohamed El-Sayed MANSOUR, Hammed Ahmed Mohamed HASSAN and Noha Amaly Mohammed HASSAN (2014), Journal of Research \& Developments in Chemistry, DOI: $10.5171 / 2014.351498$ 


\section{Thermogravimetric analysis (TGA)}

Thermal analysis was performed by using Shimadzu TGA-50 Analyzer (Shimadzu Co. Ltd., Kyoto, Japan). Specimen (10 mg) were thermally scanned from 25 to $900^{\circ} \mathrm{C}$ in the open corundum crucibles under nitrogen with a flow rate of $\approx 200 \mathrm{ml} / \mathrm{min}$ ) at a scanning rate $10^{\circ} \mathrm{C} / \mathrm{min}$.

\section{Scanning Electron Microscopy (SEM)}

Microstructure and topography of $\mathrm{CMCH}$ and CMCH-g-PAM films were characterized by a JEOL JSM-6360 LA scanning electron microscope (JEOL Ltd., Rigaku, Tokyo, Japan). Examination was performed at an acceleration voltage of $15 \mathrm{kV}$ with a magnification power of 1000-3000X. Film samples were first coated with gold using JFC-1100E sputtering coater.

\section{Swelling measurement}

Definite weights (2 gm) of $\mathrm{CH}, \mathrm{CMCH}$ or $\mathrm{CMCH}-\mathrm{g}$-PAM were put onto sintered glass funnel, immersed in $1000 \mathrm{ml}$ beaker filled with distilled water and then covered with Para-film. Afterwards, water was withdrawn by water pump throughout constant time of $15 \mathrm{~min}$, and then weight the swollen samples. Weight values were taken at equilibrium, which was determined by three consecutive measurements having the same weight. The swelling ratio of the specimens was calculated according to the following equation:

$$
S_{R}=\frac{w_{s}-W_{d}}{w_{d}}
$$

Where Ws and $W_{d}$ represented the weights of the swollen and dry state samples, respectively. The dynamic weight gain was determined versus time to illustrate the swelling behavior of these polymers.

\section{Flocculants performance assessment (Jar Test)}

To prepare the clay suspension (turbid water), accurate weighed amounts (5 - $20 \mathrm{gm}$ ) of kaolinite powder were dispersed in distilled water with stirring using mixer at 500 rpm for 2 min. Adjusting pH value (4-9) during stirring was achieved using $0.1 \mathrm{~N}$ $\mathrm{NaOH}$ or HCL. Then stirring continues for another $2 \mathrm{~min}$ at a rate of $100 \mathrm{rpm}$. Temperature keeps in water bath at constant degree, with adding $\mathrm{NaCl}$ to obtain solution with ionic strength ranging from 0.1 to 0.6 M. Afterwards, allow the dispersed solutions to settle for 1 - $20 \mathrm{~min}$., before sampling using automatic pipette from a height of $2 \mathrm{~cm}$ below the surface in each beaker for turbidity measurement. For assessing flocculants' efficiency, control (without flocculants) was first used to measure the initial turbidity $\left(\mathrm{T}_{0}\right)$, and so, the turbidity of the clay suspension after adding different amounts of the flocculants $(1-10 \mathrm{ppm})\left(\mathrm{T}_{\mathrm{t}}\right)$ was measured using the same sampling method. The turbidity removal percent was calculated by the following equation;

$$
\text { Turbidity removal } \%=\frac{T_{s s}-T_{t}}{T_{p}}
$$

\section{Results and Discussion}

\section{Factors affecting grafting percentage}

\section{CMCH concentration}

Generally, grafting percentage $(\% \mathrm{G})$ was found to increase significantly with increasing $\mathrm{CMCH}$ concentration as shown in Figure 1 , where $\% \mathrm{G}$ was 66 at $\mathrm{CMCH}$ concentration of $0.3 \%$, however it reached its maximum $(146 \%)$ at $\mathrm{CMCH}$ concentration of $2 \%$. Whereas, further increase in $\mathrm{CMCH}$ concentration led to a significant decrease in grafting percentage which reached to $18 \%$ at $\mathrm{CMCH}$ concentration of $24 \%$. The initial increase in the grafting percentage associated with the increase of carboxymethyl chitosan concentration can be attributed to the increase in the number of active sites (amine and hydroxyl groups) on carboxymethyl chitosan molecules leading to accommodate

Emad Ali SOLIMAN, El-Sayed Mohamed El-Sayed MANSOUR, Hammed Ahmed Mohamed HASSAN and Noha Amaly Mohammed HASSAN (2014), Journal of Research \& Developments in Chemistry, DOI: $10.5171 / 2014.351498$ 
higher amounts of acrylamide monomer (AM) and consequently increase the produced grafted PAM (scheme 1). Whereas, further increase in $\mathrm{CMCH}$ concentration may increase the viscosity of the reaction medium that can cause hindrance of the reactants' collision and thus reducing their reactivity, in addition to a decrease of $\mathrm{AM} / \mathrm{CMCH}$ ratio. These findings were in agreement with those obtained in the study of Ranjbar-Mohammadi with his colleagues on grafting chitosan onto wool fabric [26].



Scheme 1: CMCH grafting mechanism

Emad Ali SOLIMAN, El-Sayed Mohamed El-Sayed MANSOUR, Hammed Ahmed Mohamed HASSAN and Noha Amaly Mohammed HASSAN (2014), Journal of Research \& Developments in Chemistry, DOI: $10.5171 / 2014.351498$ 


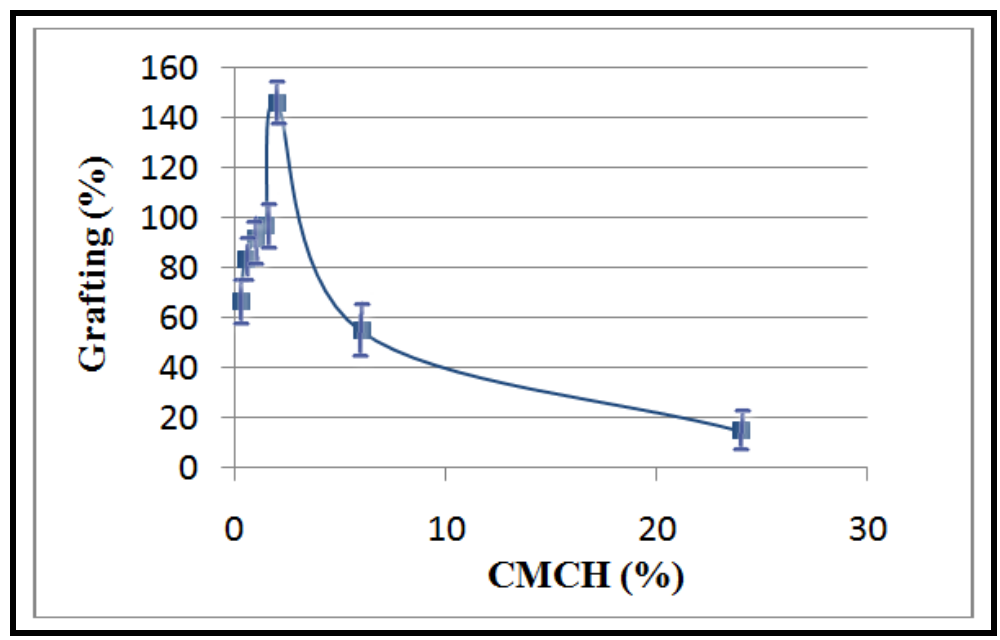

Figure 1: Effect of $\mathrm{CMCH}$ concentration on grafting percentage

\section{Monomer concentration}

The grafting percentage grew dramatically with increasing AM concentration reaching its maximum value at AM concentration of 6 $\%$ (Figure 2). However, higher increase in AM concentration led to a noticeable decrease of the grafting percentage. These results were not in agreement with the results reported by Ávila with his workers [7]. This

behavior could be explained by the fact that an increase of AM concentration leads to the accumulation of monomer molecules in close to the $\mathrm{CMCH}$ backbone. Hence, increasing monomer concentrations over saturation could be in favor of free radical-induced polymerization of AM monomer on expense of its reaction with $\mathrm{CMCH}$ polymer backbone.

i.e., homopolymerization overlies on grafting copolymerization. Furthermore, the formation of homopolymer in the presence of an excess of monomer can shield the polymer backbone and consequently stands as a hurdle towards graft copolymerization. Such assumptions can be associated with diffusion limitations, which can be governed by the dilution [26].

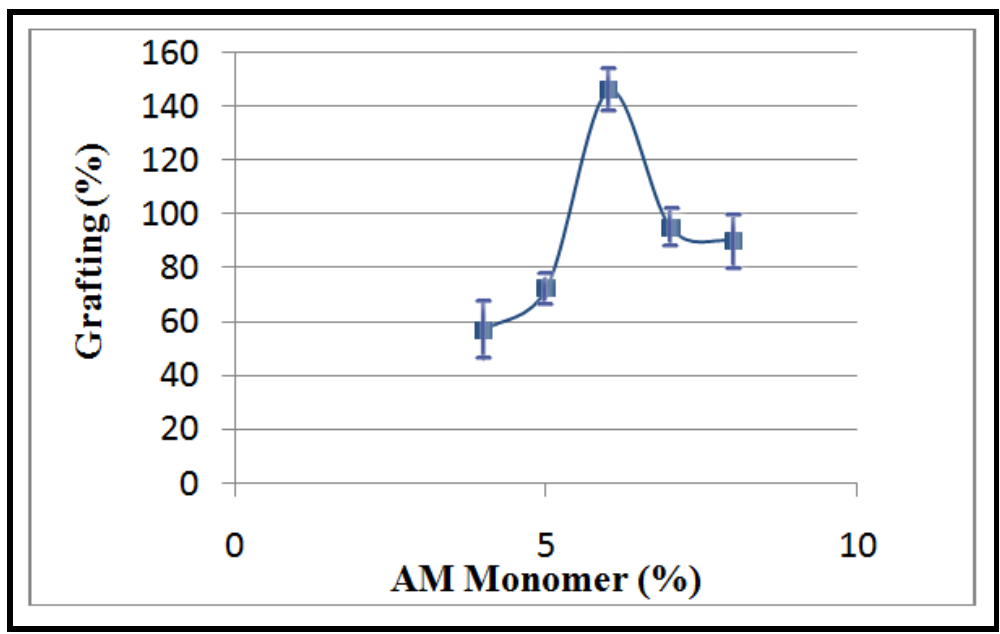

Figure 2: Effect of monomer concentration on grafting percentage

Emad Ali SOLIMAN, El-Sayed Mohamed El-Sayed MANSOUR, Hammed Ahmed Mohamed HASSAN and Noha Amaly Mohammed HASSAN (2014), Journal of Research \& Developments in Chemistry, DOI: $10.5171 / 2014.351498$ 


\section{Initiator concentration}

Indeed, initiator concentration is well known to have a crucial impact on polymerization. Therefore, determining the optimum concentration of PPS in view of the enhancement of grafting was achieved by using concentrations in the range of $0.05-1 \%$ at constant concentrations for both $\mathrm{CMCH}$ and AM. The obtained results were presented in Figure 3. These results indicated that the grafting percentage increased with increasing the initiator concentration up to $0.1 \%$, thereafter it decreased gradually with the further increase in PPS concentration. The increase of grafting percentage can be attributed to the increase of active grafting sites on the $\mathrm{CMCH}$ backbone chains (free radicals) with the increase of initiator concentration which in turn led to higher grafting. While, its decrease at PPS concentration over $0.1 \%$ can be explained on the basis that excessive free radicals produced simultaneously at these high concentrations could activate multiple free radicals coupling that led to the formation of AM homopolymer or cross-linking $\mathrm{CMCH}$ since free radicals formed onto $\mathrm{CMCH}$ chains can be terminated prior to AM addition [28]. Hence, the formation of homopolymers can be expected due to the non-availability of active sites on $\mathrm{CMCH}$. On the other hand, at higher initiator concentration, PPS can decompose to $\mathrm{HSO}_{4}{ }^{\circ}$ and $\mathrm{O}_{2}$ whilst $\mathrm{O}_{2}$ act as scavenger for free radical formed on polymer backbone chains and thus decreasing the grafting polymerization [29].

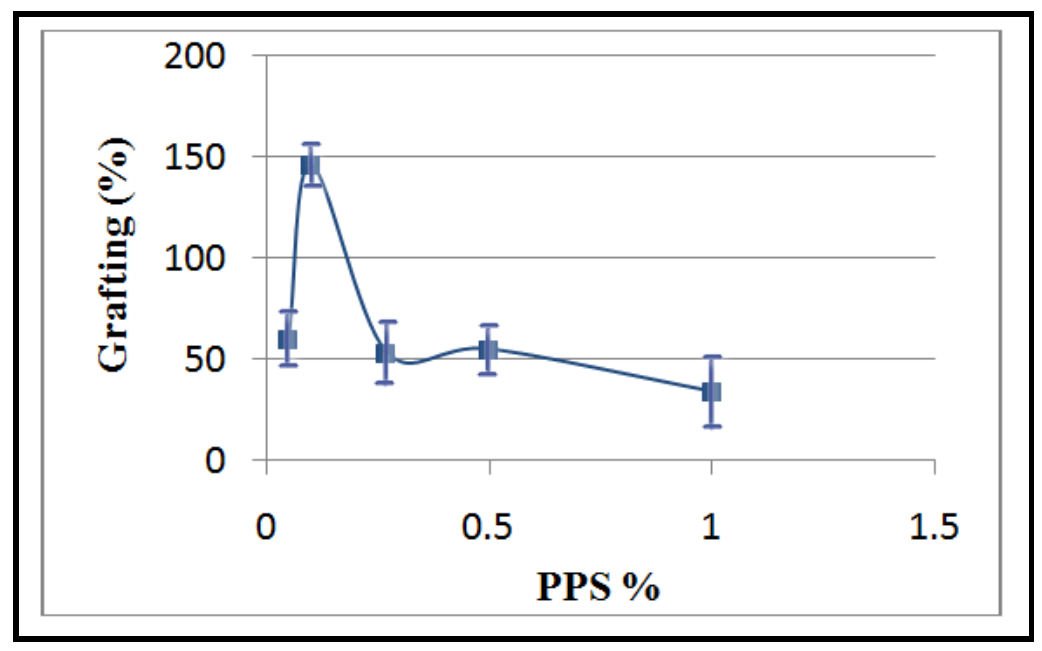

Figure 3: Effect of initiator concentration on grafting percentage

\section{Grafting temperature}

As shown from Figure 4 when grafting copolymerization was accomplished at varying temperature in the range of $45-65^{\circ} \mathrm{C}$, highest grafting percentage was attained at $55^{\circ} \mathrm{C}$, while it was decreased at lesser or upper optimum temperature. These findings can be explained in view of the fact that the low temperatures are insufficient to activate free radicals' formation. However, obtaining the highest grafting percentage at optimum temperature can be ascribed to its ability to promote formation of free radicals and swelling of $\mathrm{CMCH}$ leading to enhance diffusion of AM monomer. The further increase in temperature can be in favor of the combination of macro-radicals to be dominant reactions leading to the formation of $\mathrm{AM}$ homopolymer which increases the viscosity of the reaction medium and thereby hinders for the diffusion of monomer and inhibits graft copolymerization [30]. Based on the aforementioned, the optimum temperature meets the halfway between both tendencies, since it facilitates the diffusion of AM mono-

Emad Ali SOLIMAN, El-Sayed Mohamed El-Sayed MANSOUR, Hammed Ahmed Mohamed HASSAN and Noha Amaly Mohammed HASSAN (2014), Journal of Research \& Developments in Chemistry, DOI: $10.5171 / 2014.351498$ 
mer into $\mathrm{CMCH}$ phase and activates the formation of free radicals via increasing the dissociation rate of initiator to be in the active form [29].

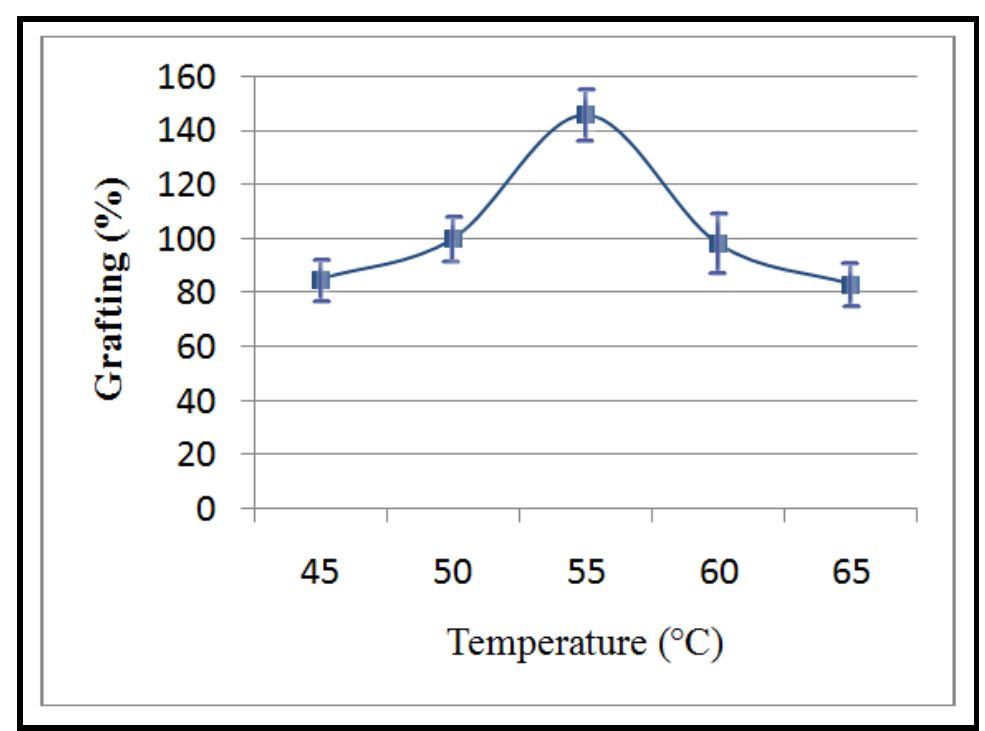

Figure 4: Effect of temperature on grafting percentage

\section{Grafting time}

The effect of the reaction time on the grafting percentage was presented in Figure 5. The grafting percentage was gradually increased with extending the reaction time from 1 to 3 hrs. Nevertheless, it decreased with the prolongation of the reaction time over 3 hrs. These findings are in agreement with those of Joshi and Sinha that reported that there is a slight decrease in the grafting percentage with the increasing time of polymerization, and thereafter it becomes constant [22]. The increase of the grafting percentage with the prolonging time of the reaction can be attributed to give a break to produce more free radicals onto polymer backbone and open up its structure. Forasmuch, the bulky groups, such as carboxmethyl $\left(-\mathrm{CH}_{2} \mathrm{COOH}\right)$ in the $\mathrm{CMCH}$ can promote the swelling and thereby diffusion of PPS and AM into $\mathrm{CMCH}$ [30]. However, the observed decrease in the grafting percentage with the long time of reaction can be explained on the basis of depleting acrylamide monomer with the progression of the grafting process. Furthermore, this decrease may be also attributed to enhance the viscosity of the reaction medium by growing the yield of graft copolymers and subsequently slow down reactants' collision and lower grafting ratios [29]. 


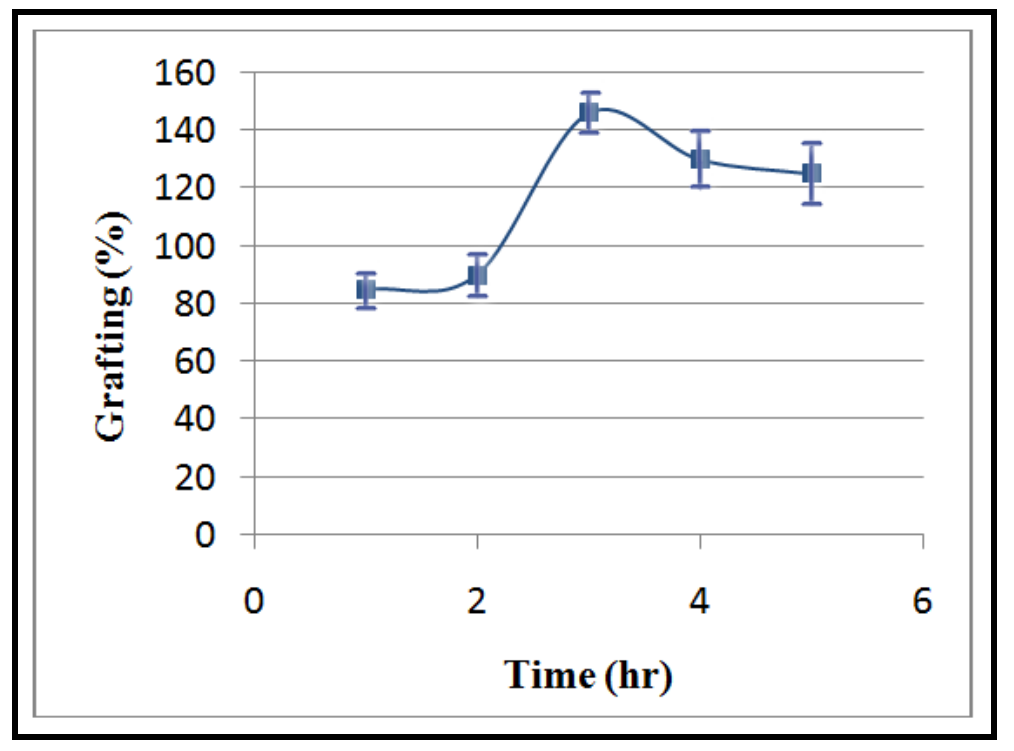

Figure 5: Effect of grafting time on grafting percentage

\section{Physico-chemical properties characteri- zation}

\section{Compositional properties}

The spectra of $\mathrm{CH}$ and grafted polymer (CMCH-g-PAM) were shown in Figure 6. FTIR spectrum of chitosan exhibited two strong characteristic absorptions peaks; one $\sim 1575$ $\mathrm{cm}^{-1}$ due to $\mathrm{N}-\mathrm{H}$ bending and other peak assigned to $\mathrm{N}-\mathrm{H}$ and $\mathrm{O}-\mathrm{H}$ stretching at $\sim 3369$ $\mathrm{cm}^{-1}$. Furthermore, adsorption peak appeared at $1321 \mathrm{~cm}^{-1}$ due to $\mathrm{C}-\mathrm{N}$ bending, in addition to a small band at $1649 \mathrm{~cm}^{-1}$ which refers to $(\mathrm{C}=0)$ of acetamide group (-CONH-) belonging to acetylated unites of chitosan. However, FTIR spectrum of CMCH-g-PAM exhibited an absorption band at $\sim 1662 \mathrm{~cm}^{-1}$ due to $\mathrm{C}=0$ (amide) stretching vibrations, peak at $\sim 1423 \mathrm{~cm}^{-1}$ of $\mathrm{COO}$ stretching vibrations and peak at $\sim 2945 \mathrm{~cm}^{-1}$ due to the $\mathrm{C}-\mathrm{H}$ of $\mathrm{CH}_{2}$ stretching vibrations. Such graft copolymer spectrum also showed more intense broad adsorption peak appeared at $\sim 3390 \mathrm{~cm}^{-1}$ due to the overlapping of $\mathrm{O}-\mathrm{H}$ and $\mathrm{N}-\mathrm{H}$ stretching of $\mathrm{CMCH}$ and PAM.

The slight shifting and more intensityof the latter absorption peak confirm grafting reac

tion between carboxymethyl chitosan and polyacrylamide.

Emad Ali SOLIMAN, El-Sayed Mohamed El-Sayed MANSOUR, Hammed Ahmed Mohamed HASSAN and Noha Amaly Mohammed HASSAN (2014), Journal of Research \& Developments in Chemistry, 


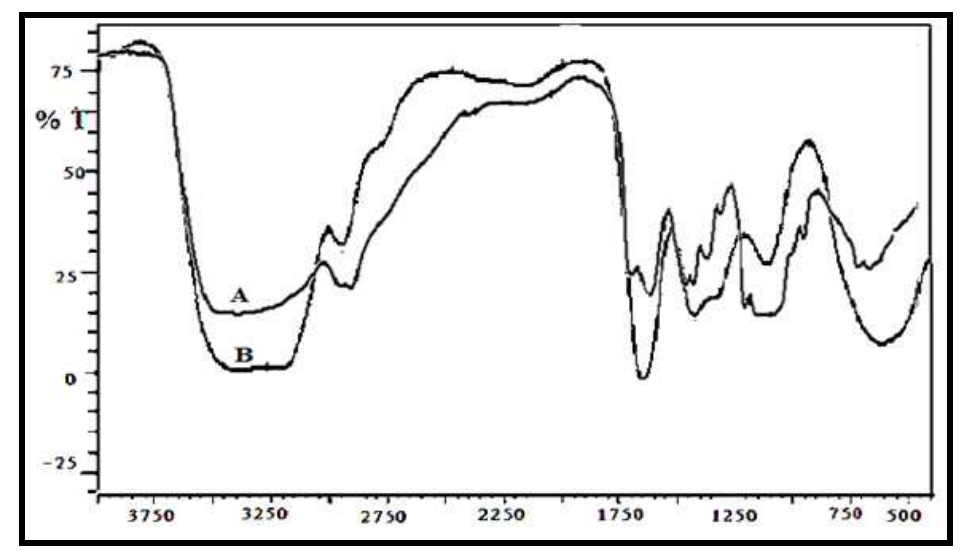

Figure 6: FTIR spectra of Chitosan (A) and CMCH-g-PAM copolymer (B)

\section{Thermal stability}

Thermal stability of $\mathrm{CH}$ and $\mathrm{CMCH}-g$-PAM were assessed by thermogravimetric analysis. Figure 7 presented the thermograms of chitosan and graft copolymer. The thermogram of chitosan exhibited four distinct stages of thermal degradation; weight loss $13.8 \%$ in the range of $27-152{ }^{\circ} \mathrm{C}$ was corresponding to the loss of adsorbed and bound water. Since chitosan contains $\left(\mathrm{NH}_{2}\right)$ and $(\mathrm{OH})$ functional groups, the hydrogen bonding force is strongly formed among molecules [31]. The second stage of the thermal degradation occurred at $220-455$ ${ }^{0} \mathrm{C}$ with weight loss was $\sim 21 \%$. This weight loss can be attributed to degradation and deacetylation of chitosan [32]. Further weight loss by $\sim 20 \%$ occurred at temperature range $455-800{ }^{\circ} \mathrm{C}$ due to the dehydration of glycoside unit and decomposition of the acetylated and deacetylated units of chitosan. On the other side, CMCH-g-PAM exhibited also four subsequent distinct degradation (weight loss) stages $\left(25-799{ }^{\circ} \mathrm{C}\right)$; first stage was from $25-168{ }^{\circ} \mathrm{C}$ with weight loss $\sim 16 \%$ due to the loss of adsorbed and bound water, second stage $228-352{ }^{\circ} \mathrm{C}$ during which $\sim 17 \%$ weight loss was assigned to decarboxylation of $\mathrm{CMCH}$ and subsequent loss of $\mathrm{CO}_{2}$. Afterwards, $\sim 26 \%$ weight loss between $354-472{ }^{\circ} \mathrm{C}$ that contributes to the degradation of both the $\mathrm{CMCH}$ backbone and the grafted polyacrylamide chains amalgamated onto CMCH-g-PAM. Finally $\sim 22 \%$ weight loss up to $799{ }^{\circ} \mathrm{C}$ due to the decomposition of the glycosidic units of $\mathrm{CMCH}$. These findings revealed that grafting slightly increased the initial decomposition temperature of CMCH- $g$-PAM compared with that for chitosan.

Emad Ali SOLIMAN, El-Sayed Mohamed El-Sayed MANSOUR, Hammed Ahmed Mohamed HASSAN and Noha Amaly Mohammed HASSAN (2014), Journal of Research \& Developments in Chemistry, DOI: $10.5171 / 2014.351498$ 


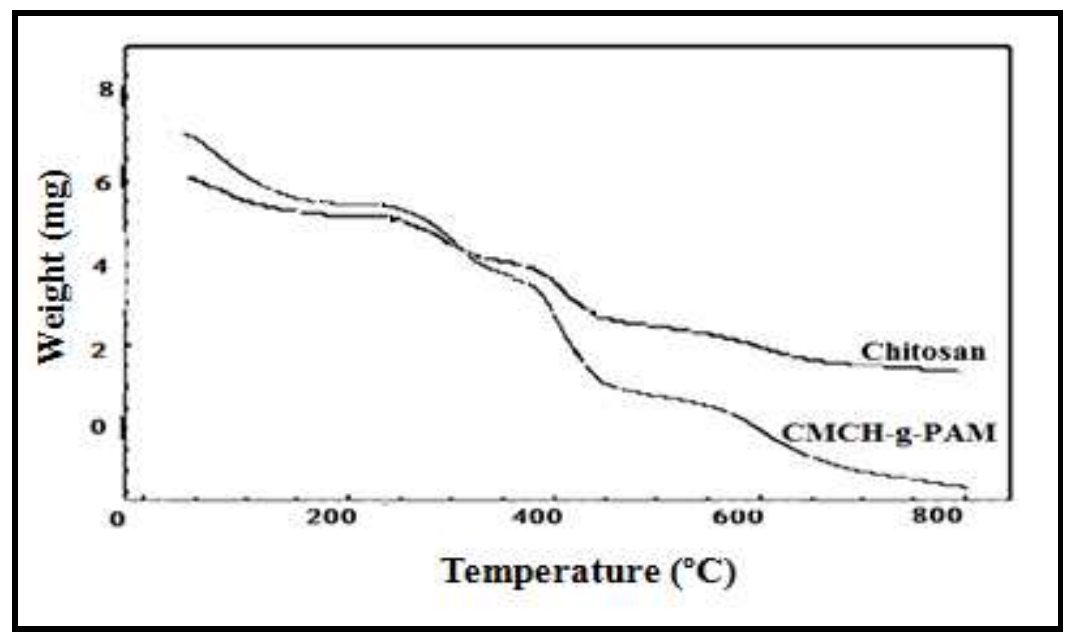

Figure 7: Thermograms of Chitosan and CMCH-g-PAM copolymer

\section{Morphological and micro-structure prop- erties}

Scanning electron micrographs of carboxymethyl chitosan and its graft copolymer with PAM were shown in Figure 8. Figure 8A exhibited cohesive and dense structure of $\mathrm{CMCH}$ via appearing relatively smooth and continuous surface with no distinct perturbations. Whereas, SEM micrograph of CMCH-g-PAM (Figure 8B) showed that the graft copolymerization of chitosan with polyacrylamide dramatically changed the conformation of $\mathrm{CMCH}$ polymer chains, since the heterogonous structure was observed because the interruption of the chain packing occurs by conformational defects leading to major disruption in the perfection of the chain packing. These conformational defects in $\mathrm{CMCH}$ chains can be attributed to the grafted PAM chains and consequently confirms graft polymerization.



Figure 8: Scanning electron micrographs (SEM) of CMCH (A) and CMCH-g-PAM copolymer (B)

Emad Ali SOLIMAN, El-Sayed Mohamed El-Sayed MANSOUR, Hammed Ahmed Mohamed HASSAN and Noha Amaly Mohammed HASSAN (2014), Journal of Research \& Developments in Chemistry, DOI: $10.5171 / 2014.351498$ 


\section{Swelling behavior}

Swelling of $\mathrm{CH}, \mathrm{CMCH}$ and $\mathrm{CMCH}-\mathrm{g}-\mathrm{PAM}$ was gravimetrically determined by measuring the weight gain with time when they were placed in distilled water at room temperature. Measurements were taken at equilibrium, which was determined by three consecutive measurements having the same weight. Chitosan and CMCH gave swelling by 40 and $\sim 50 \%$, respectively at $15 \mathrm{~min}$, after which these polymers were going to dissolve.
However, swelling of grafted copolymer reached to $\sim 55 \%$ at $30 \mathrm{~min}$. (Figure 9). Change swelling behavior of chitosan may be attributed to the introduction of carboxymethyl groups that improve remarkably the solubility of $\mathrm{CMCH}$ in aqueous solution [33]. However, hydrogen bonding is responsible for enhancing moisture retention ability of graft copolymer, where, the hydrophilic groups such as carboxylic and amino groups is known to form $\mathrm{H}$-bonds with water molecules.

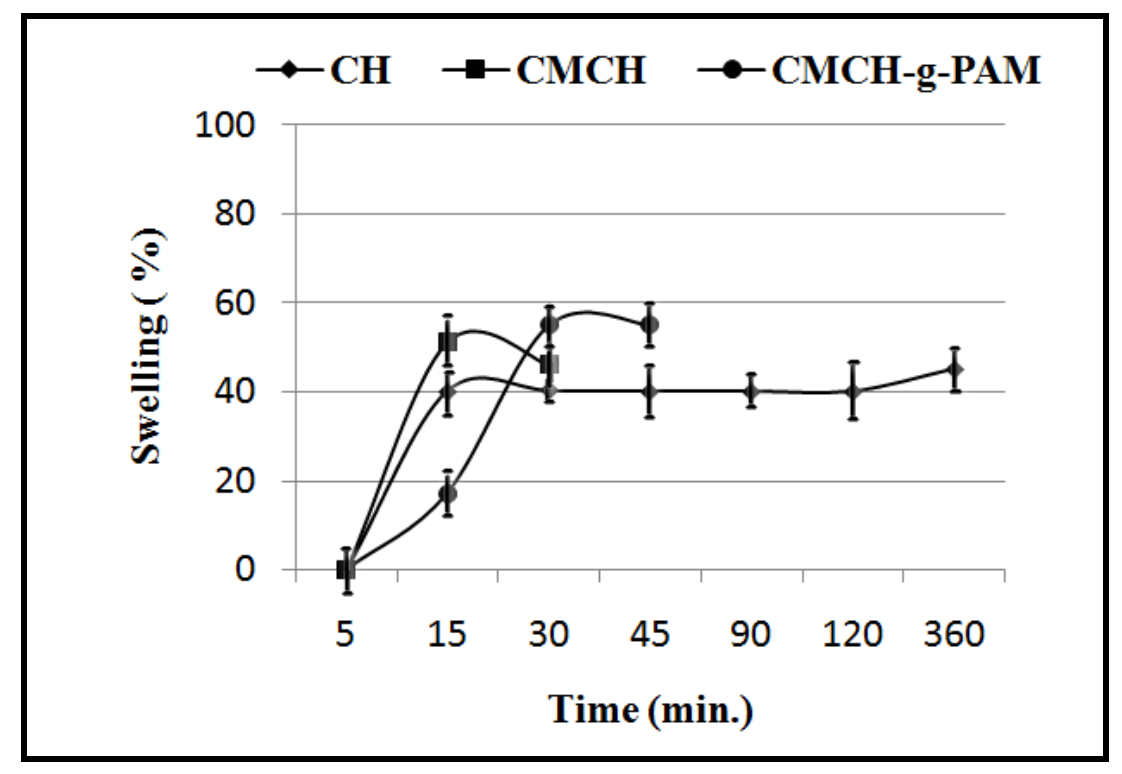

Figure 9: Swelling percentages of chitosan, $\mathrm{CMCH}$ and $\mathrm{CMCH}-\mathrm{g}-\mathrm{PAM}$ vs. time

\section{Factors affecting flocculation (turbidity removal of clay suspensions)}

\section{Polymer type}

The compositional characteristics of the polymer-based flocculants including both type and content of functional groups, branching, and molecular weight of the polymer are considered to be among the main factors affecting the flocculation efficiency. Therefore, the flocculation performance of $\mathrm{CH}, \mathrm{CMCH}, \mathrm{PAM}$, and $\mathrm{CMCH}-\mathrm{g}-\mathrm{PAM}$ was investigated by measuring the turbidity of kao- linite suspensions after flocculation (Figure 10). From these results, it was observed that CMCH-g-PAM grafted copolymer has the highest flocculation efficiency. On the other hand, chitosan revealed the lowest efficiency. However, the efficiency of $\mathrm{CMCH}$ was comparable to that of PAM. High flocculation performance of graft copolymer can be due to their loose and branched structures giving potential flexible behavior that enhances the bridging effect of these flocculants. Additionally, charge density arising from various charged functional groups $\left(-\mathrm{COO},-\mathrm{NH}_{2}\right)$ on these flocculants provides flocculation power

Emad Ali SOLIMAN, El-Sayed Mohamed El-Sayed MANSOUR, Hammed Ahmed Mohamed HASSAN and Noha Amaly Mohammed HASSAN (2014), Journal of Research \& Developments in Chemistry, DOI: $10.5171 / 2014.351498$ 
towards fine dispersed kaolinite particulates. This was on contrary of $\mathrm{CH}, \mathrm{CMCH}$, and PAM which are considered linear polymers.

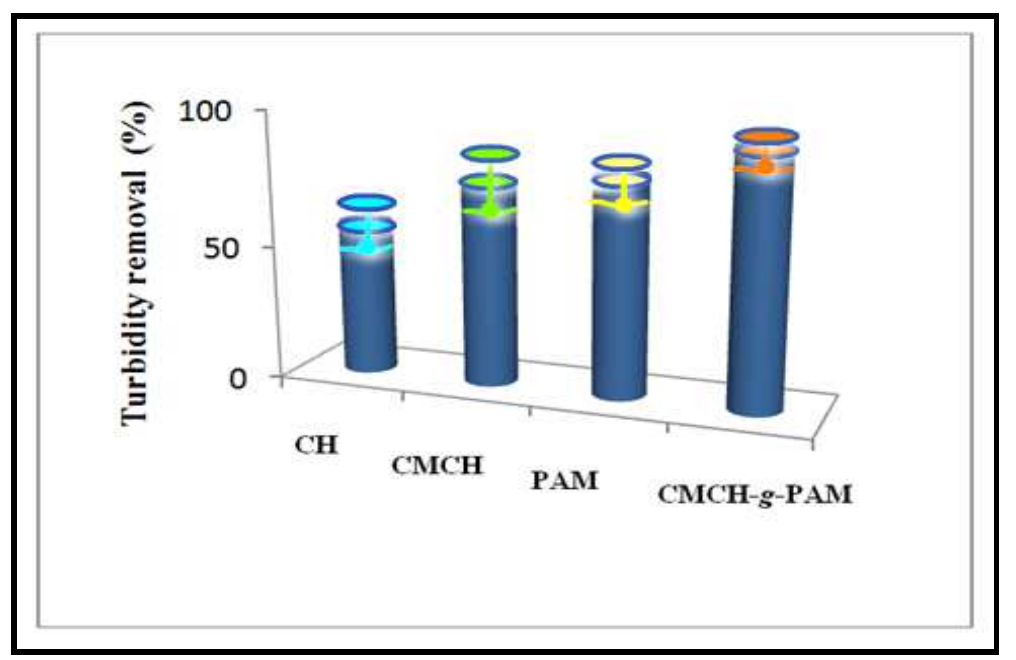

\section{Figure 10: Turbidity removal of clay suspension using chitosan, CMCH, PAM and CMCH- $g$-PAM}

\section{pH}

In the present study, the initial turbidity of kaolinite suspensions (turbid water) was measured at different $\mathrm{pH}$ values. These measurements indicated that the initial turbidity was increased by increasing $\mathrm{pH}$ as presented in Figure 11. This can be due to the increase of - ve charge density on kaolinite particulates in alkaline media (at basic $\mathrm{pH}$ 9) that increase repulsion between clay particles together and with -ve hydroxyl groups of basic medium which prevent aggregation of the particles. On the contrary, the highest percentages of turbidity removal from clay suspensions using $\mathrm{CH}, \mathrm{CMCH}$ and $\mathrm{CMCH}-\mathrm{g}$-PAM were observed in acidic medium $(\mathrm{pH}=6)$ (Figure 12). This result can be attributed to increasing $+v$ e charge density on $\mathrm{CH}, \mathrm{PAM}, \mathrm{CMCH}$ and $\mathrm{CMCH}-\mathrm{g}$-PAM as a result to protonation of amine groups that form $+v e$ charge centres on these polymer molecules, whereas, kaolinite particles have - ve zeta potential in this acidic medium and consecutively so strong electrostatic attraction has been achieved between oppositely charged macromolecular flocculants and clay particles (Charge neutralization). However, the superior flocculating efficiency of $\mathrm{CMCH}-g$-PAM and $\mathrm{CMCH}$ compared with $\mathrm{CH}$ can refer to higher charge density arising from many of amine groups carrying a positive charge at the acidic $\mathrm{pH}$ and thus the hydrophilicity and wettability of these polymer molecules were increased. Furthermore, high molecular weight and branching of graft copolymer enhance the flocculation performance since the extended chain tends to be adsorbed onto the particle in a flat configuration, whereas the negative charges on the bare parts of surface of one particulate easily attach to the excess net residual positive charges of the polymer absorbed on another one $[34,35]$. On the other side, CMCH- $g$-PAM accomplished only about $75 \%$ of turbidity removal at neutral $\mathrm{pH}$ as shown in Figure 12. Such lower efficiency of turbidity removal occurring at pH 7 compared with that occurring at acidic $\mathrm{pH}$ can be attributed to that at this neutral $\mathrm{pH}$, kaolinite zeta potential becomes more $-v e$, whilst less protonation of amine groups leads to lower + ve zeta potential of flocculants than that occurring in acidic medium and consequently attraction force between clay particulates and polymer can be reduced. In alkaline medium $(\mathrm{pH}=9)$, the

Emad Ali SOLIMAN, El-Sayed Mohamed El-Sayed MANSOUR, Hammed Ahmed Mohamed HASSAN and Noha Amaly Mohammed HASSAN (2014), Journal of Research \& Developments in Chemistry, DOI: $10.5171 / 2014.351498$ 
turbidity removal percentage of grafted copolymer decreased to about $37 \%$. This can be due to - ve zeta potentials for both carboxylate groups of graft copolymers and ka- olinite particulates and simultaneously less protonation of amine groups, therefore the resultant electrostatic repulsion can reduce the flocculating performance $[6,36]$.

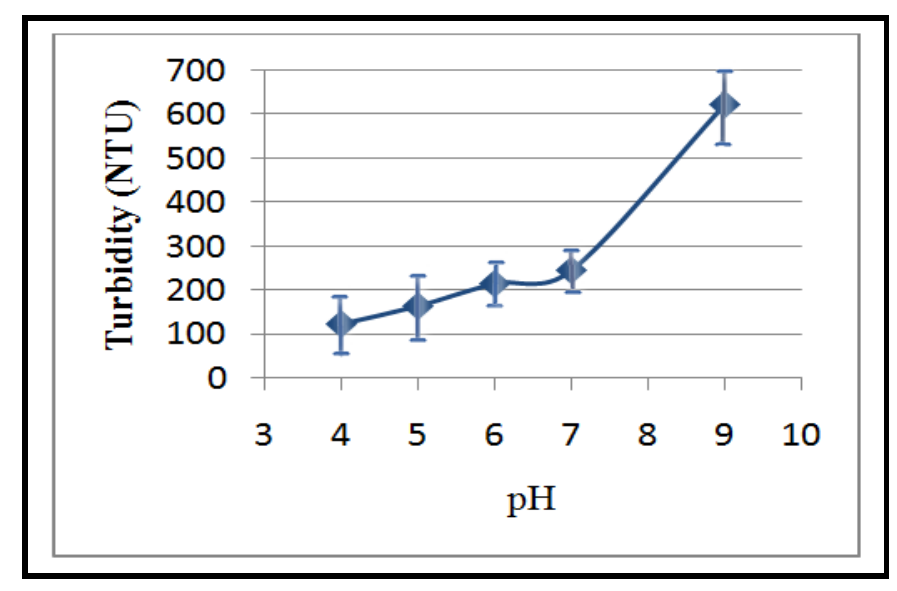

Figure 11: Effect of $\mathrm{pH}$ on the initial turbidity of clay suspension $(10 \mathrm{gm} / \mathrm{L})$

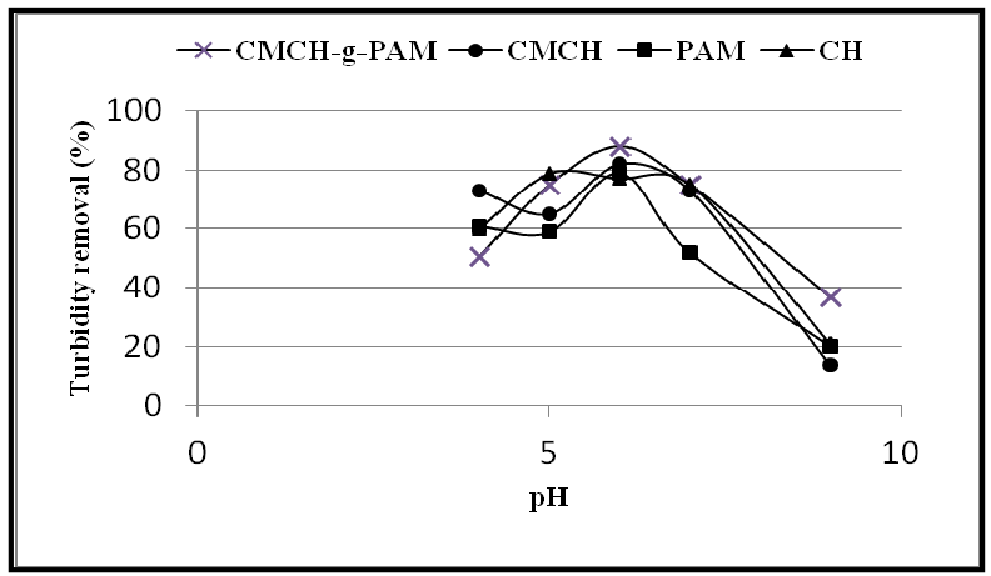

Figure 12: Effect of pH of clay suspension (10 gm/L) on turbidity removal using flocculants at dosage of 5 ppm

\section{Grafting percentage}

The grafting percentage of CMCH- $g$-PAM exhibited a noticeable effect on the flocculation efficiency (Figure 13). In general, turbidity removal percentage for clay suspension using graft copolymer was noticeably increased with increasing the grafting percentage, so the graft copolymer having the highest grafting percentage (146\%) exhibited effi- ciency of the turbidity removal that reached $90 \%$ with a noticeable mechanical stability for the resultant flocs (Figure 14). Since these expanded polymer chains play an axial role in bridging mechanisms that are highly responsible for flocculation performance. These results can be explained on the basis that increasing grafting ratio was associated with increasing the molecular weight of polymers and the length of the grafted poly-

Emad Ali SOLIMAN, El-Sayed Mohamed El-Sayed MANSOUR, Hammed Ahmed Mohamed HASSAN and Noha Amaly Mohammed HASSAN (2014), Journal of Research \& Developments in Chemistry, DOI: $10.5171 / 2014.351498$ 
mer chains that led to enhancing flocculating performance via bridging mechanism. On the other hand, positive charges of protonated amine groups $\left(\mathrm{NH}_{3}{ }^{+}\right)$mounted onto PAM long branched chains linked to the $\mathrm{CMCH}$ backbone, whilst these $\mathrm{CMCH}$ backbone chains carry negative charge on anionic carboxylate groups $\left(-\mathrm{COO}^{-}\right)$when dissociate at pH 6 enhancing the flocculation performance via charge neutralization mechanism. Hence, the adjacent charged groups that repel each other will lead to expanding the polymer chains. Therefore, the bridging by high molecular weight polymer chains can be considered the main mechanism in the flocculation of kaolinite particulates with the contribution of role of ionic functional groups in charge of neutralization, because increasing the degree of ionicity (charge density) of the more expanded form of polymer chains leads to enhancing flocculating power with increasing the rate of settling, i.e., increasing of the floc size [37].

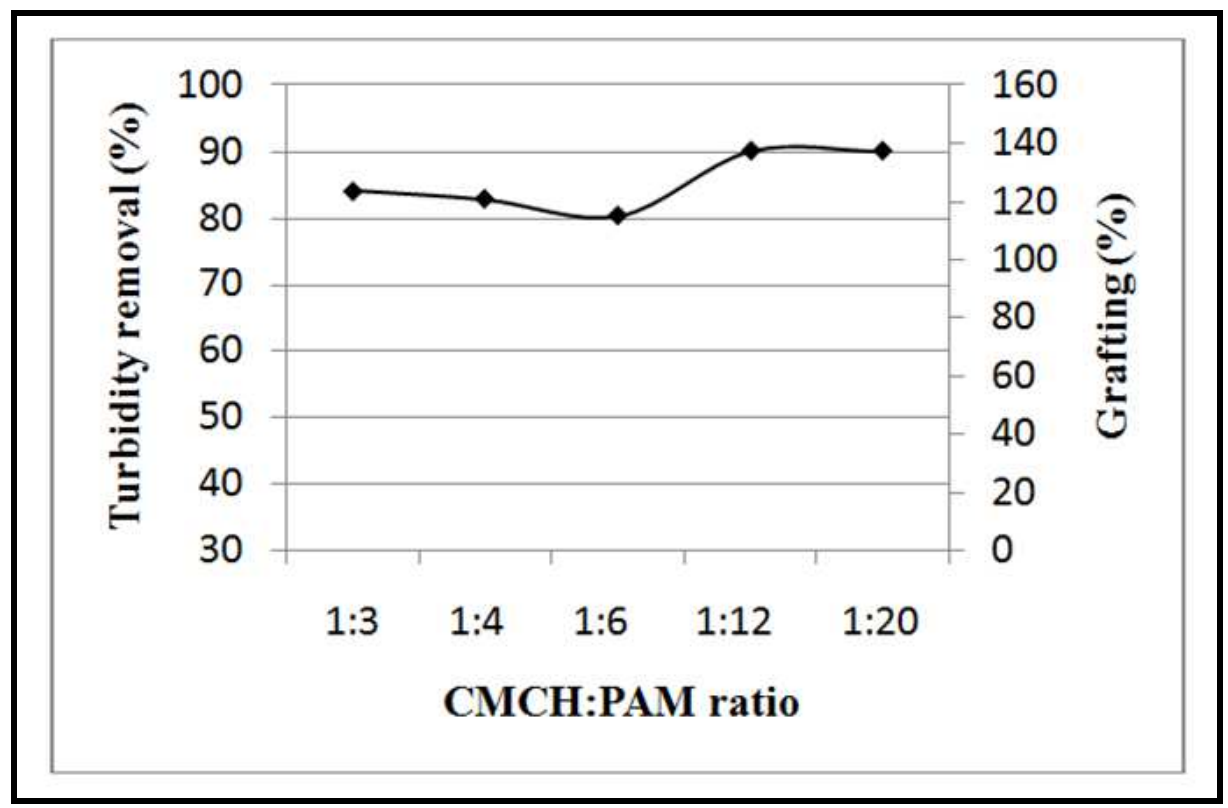

Figure 13: Effect of grafting percentage on turbidity removal from clay suspension $(10 \mathrm{gm} / \mathrm{L})$ at pH 6 using CMCH-g-PAM at dosage of 5 ppm

Emad Ali SOLIMAN, El-Sayed Mohamed El-Sayed MANSOUR, Hammed Ahmed Mohamed HASSAN and Noha Amaly Mohammed HASSAN (2014), Journal of Research \& Developments in Chemistry, DOI: $10.5171 / 2014.351498$ 


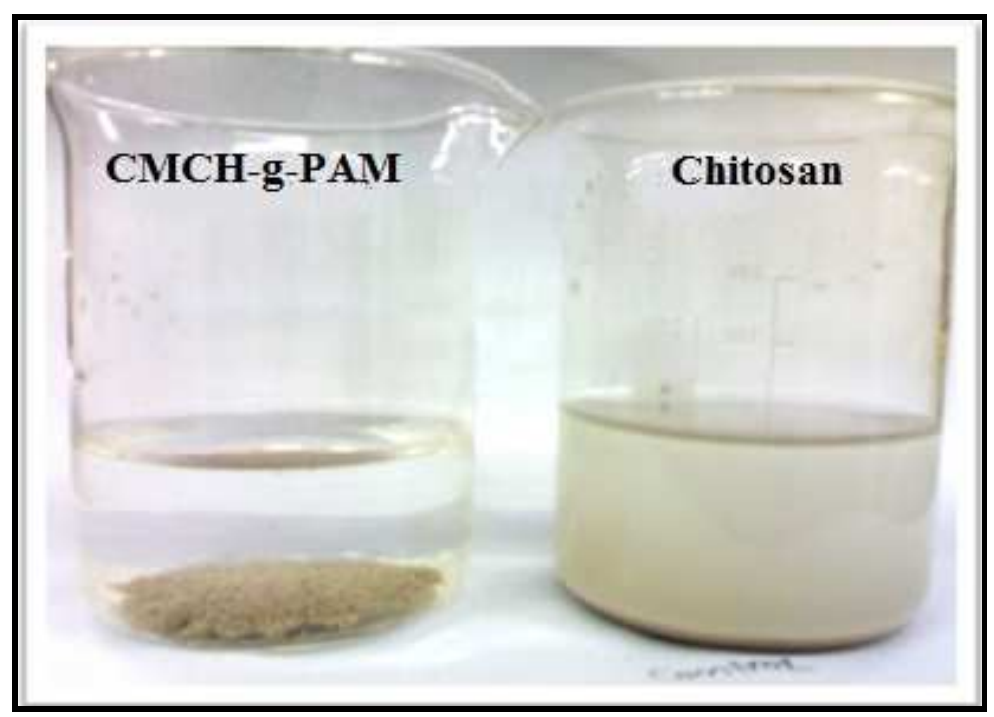

Figure 14: Photograph indicating turbidity removal from kaolinite suspension (10 g/L) using chitosan and CMCH-g-PAM (\% G = 146), at dosage of 2.5 ppm and pH 6

\section{Conclusion}

In the present study, carboxymethyl chitosan was successfully grafted with acrylamide using potassium persulphate as an initiator. Graft copolymers with high grafting percentages reached to $146 \%$ were obtained. The optimization of the parameters reaction were studied. The finding of these experi

ments indicated that performing grafting copolymerization using carboxymethyl chitosan and acrylamide at the concentration of 2 and $6 \%$, respectively in the presence of $0.1 \%$ of initiator at $55^{\circ} \mathrm{C}$ for $3 \mathrm{hrs}$ resulted in graft copolymer having the highest grafting percentage. The graft copolymers were structurally characterized using FTIR, and SEM. Moreover, the thermal stability of the graft copolymer was assessed using TGA. These analyses confirmed the formation of graft copolymer having higher initial thermal decomposition temperature. The flocculation performance of the obtained graft copolymer was tested using kaolinite suspensions, where the effect of grafting percentage and dosages of graft copolymer and $\mathrm{pH}$ on flocculation performance were studied with comparison to chitosan, $\mathrm{CMCH}$ and PAM.
Under the same conditions, CMCH-g-PAM was found to be more efficient than chitosan, carboxymethyl chitosan and polyacrylamide.

\section{Acknowledgements}

The authors highly appreciate that this work has been funded by Egyptian Academy of Scientific Research and Technology (ASRT).

\section{References}

1. Shamim, A, Kaneco, S, Ohta, K, Mizuno, T. and Kani, K. (2001)" Use of some natural and waste materials for waste water treatment," Water Research, 35 (15) 3738-3742.

2. Edeline F. (1992) L'épuration Physico-chimique des eaux :Théorie et technologie, Editions Cebedoc (éditeur), Lavoisier Technique et Documentation, 2e édition, liege, Paris.

3. Menoret C. (2001) Thèse du Doctorat en sciences de l'eau et de l'environnement continental, Université MONPELLIER II, France, 116.

4. Gregory, J. (1973) "Rates of flocculation of latex particles by cationic polymers,"

Emad Ali SOLIMAN, El-Sayed Mohamed El-Sayed MANSOUR, Hammed Ahmed Mohamed HASSAN and Noha Amaly Mohammed HASSAN (2014), Journal of Research \& Developments in Chemistry, DOI: $10.5171 / 2014.351498$ 
Journal of Colloid and Interface Science, 42 (2) 448-456.

5. Bottero, J.Y. D, Tchoubar, M. A. V, Quienne, A. P. and Fiessinger, F. (1990) "Flocculation of silica colloids with hydroxy aluminum polycations: Relation between floc structure and aggregation mechanisms, Langmuir, 6 (3) 596-602.

6. Sang, Y. and Xiao, H. (2008) "Clay flocculation improved by cationic poly (vinyl alcohol)/anionic polymer dual-component system," Journal of Colloid and Interface Science, 326 (2) 420-425.

7. Ávila, A., Pucci, G., Bierbrauer, K., López-González, M. and Strumia, M. (2012) "Study of optimization of the synthesis and properties of biocomposite films based on grafted chitosan," Journal of Food Engineering, 109 (4) 752-761.

8. Biswal, D. R. and Singh, R. P. (2004) "High performance flocculating agents and viscosifiers based on hydrolysed polyacrylamide grafted amylopectin and polyacrylamide grafted carboxymethyl cellulose," Carbohydrate Polymers, 57 (4) 379-387.

9. Kim, S. K. and Mendis, E. (2006)" Bioactive compounds from marine processing byproducts: A review," Food Research International, 39 (4) 383-393.

10. Kim, S. K. and Rajapakse, N. (2005)” Enzymatic production and biological activities of chitosan oligosaccharides (COS): A review," Carbohydrate Polymers, 62 (4) 357-368.

11. Shahidi, F. and Abuzaytoun, R. (2005)"Chitin, chitosan, and coproducts: chemistry, production, applications, and health effects," Advances in Food and Nutrition Research, 49, 93-135

12. Venkatesan, J. and Kim, S. K. (2010)" Chitosan composites for bone tissue engi

13. neering -An overview," Marine Drugs, 8 (8) 2252-2266.
14. Venkatesan, J, Pangestuti, R, Qian, Z. J, Ryu, B. and Kim, S. K. (2010)"Biocompatibility and alkaline phosphatase activity of phosphorylated chitooligosaccharides on the osteosarcoma MG63 cell line," Journal of Functional Biomaterials, 1(1) 3-13.

15. Meyer, H., Butte, W. and Schlaak, M. (2000)"Chitosan in wastewater treatment". Advances in Chitin Science, 4, ( ) 153-158.

16. Sudha. P. N. (2010) Chitin/chitosan and derivatives for wastewater treatment, Chitin, Chitosan, Oligosaccharides and Their Derivatives. Se-Kwon Kim (ed), CRC press. Chapter 39, 561-576.

17. Caner, H, Hasipoglu, H, Yilmaaz O. and Yilmaaz, E. (1998) "Graft copolymerization of 4 -vinylpyridine onto chitosan 1 . by ceric ion initiation," European Polymer Journal, 34 ( ) 493-497.

18. Pedram, M. Y. and Retuert, J. (1997) "Homogeneous Grafting Reaction of Vinyl Pyrrolidone onto Chitosan," Journal of Applied Polymer Science, 63 (10)1321-1326.

19. Shantha, K. L, Bala, U. and Rao, K. P. (1995) "Tailor-made chitosans for drug delivery," European Polymer Journal, 31(4) 377-382.

20. Sun, T. Xie W. and Xu, P. (2004) "Superoxide anion scavenging activity of graft chitosan derivatives," Carbohydrate Polymers, 58 (4) 379-382.

21. Srivastava, A, Tripathy, J, Mishra M. M. and Behari, K. (2007) "Modification of guar gum through grafting of 4-vinyl pyridine using potassium peroxymonosulphate/ ascorbic acid redox pair," Journal of Applied Polymer Science, 106 (2) 1353-1358.

22. Tripathy, J, Mishra, D. K, Srivastava, A, Mishra M. M. and Behari, K. (2008) "Synthesis of partially carboxymethylated guar gum-g-4-vinyl pyridine and study of its water swelling, metal ion sorption and floccula

Emad Ali SOLIMAN, El-Sayed Mohamed El-Sayed MANSOUR, Hammed Ahmed Mohamed HASSAN and Noha Amaly Mohammed HASSAN (2014), Journal of Research \& Developments in Chemistry, DOI: $10.5171 / 2014.351498$ 
tion behaviour," Carbohydrate Polymer, 72 (3) 462-472.

23. Joshi, J. M. and Sinha, V. K. (2007) Ceric ammonium nitrate induced grafting of polyacrylamide onto carboxymethyl chitosan," Carbohydrate Polymers, 67 (3) 427-435.

24. Kulkarni, A. G, Mathur, R. M. and Tandon, R. (2006) "Some chemical aspects of color removal from effluents of paper industry," Journal of Indian Pulp Paper Technical Association, 18 , 55-61.

25. Chen, X., G., Park, H., J., 2003. Chemical characteristics of O-carboxymethyl chitosans related to the preparation conditions," Carbohydrate Polymers, 53(4) 355-359.

26. Manu, B. (2007) "Physico-chemical treatment of indigo dye wastewater," Coloration Technology, 123 (3) 197-202.

27. Ranjbar-Mohammadi, M, Aramia, M, Bahramia, H, Mazaheri F. and Mahmoodi, N. M. (2010) "Grafting of chitosan as a biopolymer on to wool fabric using anhydride bridge and its antibacterial property," Colloids and Surfaces B: Biointerfaces, 76 (2) 397-403.

28. James, A. E. and Nasser, M. S. (2006) "The effect of polyacrylamide charge density and molecular weight on the flocculation and sedimentation behaviour of kaolinite suspensions," Separation and Purification Technology, 52(2) 241-252.

29. Singh, R. P. (1990) Encyclopedia of Fluid Mechanics, Gulf Publishing, Houston, Vol. 9, Chapter 14, p.p. 425-480.

30. Singh, R. P. and Tripathy, T. (2000) "High performance flocculating agent based on partially hydrolysed sodium alginate-g-polyacrylamide," European Polymer Journal, 36 (7) 1471- 1478.

31. Srivastava, A, Mishra D. K, Tripathy, J, Behari, K. and Mishra, M. M. (2008) "Graft copolymer (chitosan-g-N-vinyl formamide): Synthesis and study of its properties like swelling, metal ion uptake and flocculation," Carbohydrate Polymers, 74 (3) 632-639.

32. Radeva, T. (2001) Physical Chemistry of Polyelectrolytes, Marcel Dekker Inc., New York, USA.

33. Rey, F. A. and Varsanik, R. C. (1986) Application and function of synthetic polymeric flocculents in wastewater treatment, Water Soluble Polymers: Beauty with Performance, Glass, J. E. (ed), pp. 113-143, ACS Advances in Chemistry Series No. 213, American Chemical Society, Washington, D.C.

34. Hjerde, R. J. N, Vårum, K. M, Grasdalen, H, Tokura, S. and Smidsrød, O. (1997) "Chemical composition of O-(carboxymethyl)-chitins in relation to lysozyme degradation rates," Carbohydrate Polymers, 34 (3) 131-139.

35. Windebank, A. J, Kempen, D. H, Lewis, J. L, Lu, L. C, Yaszemski, M. J, Simha, N. X. and Wang, S. F. (2008) "Photo-cross-linked hybrid polymer networks consisting of poly (propylene fumarate) and poly(caprolactone fumarate): controlled physical properties and regulated bone and nerve cell responses," Biomacromolecules, 9 (4) 1229-1241.

36. Wirsen, A, Albertson, A. C. and Qu, X. (1999) "Structural change and swelling mechanism of pH-sensitive hydrogel based on chitosan and $D, L$ - lactic acid," Journal of Applied Polymer Science, 74, 3193-3202.

37. Yumin, D., Houbin, L., Yongmei, X., Huaiyu, Z., Kennedy, J., F. (2004) "Interactions of cationized chitosan with components in a chemical pulp suspension," Carbohydrate Polymers, 58 (2) 205-214.

38. Zhu, A, Li, L, Chan-Park, M. B. and Dai, S. (2005) "The aggregation behaviour of o-carboxymethyl chitosan in dilute aqueous solution," Colloids and Surfaces B: Biointerfaces, 43 (3-4) 143-149.

Emad Ali SOLIMAN, El-Sayed Mohamed El-Sayed MANSOUR, Hammed Ahmed Mohamed HASSAN and Noha Amaly Mohammed HASSAN (2014), Journal of Research \& Developments in Chemistry, DOI: $10.5171 / 2014.351498$ 